\title{
THE RADIO PROPERTIES OF X-RAY SELECTED EXTRAGALACTIC OBJECTS
}

\author{
L. RAMÍREZ-CASTRO, \\ I. PÉREZ-FOURNON AND F. CABRERA-GUERRA \\ Instituto de Astrofísica de Canarias
}

\section{The WGACAT Catalog of ROSAT Point Sources}

We have used the 1st revision of the WGACAT catalogue, generated from all ROSAT PSPC pointed observations from February 1991 to March 1994, available at the HEASARC public archive. This catalogue, described by White et al. (1994), contains 68,907 detections, with more than 62,000 individual sources.

The ROSAT PSPC field was divided in two parts: the first includes the center of the field roughly up to the inner support structure at 19 arcmin radius. The second is the region outside the inner support and runs from 18 to 55 arcmin. We have only considered the sources detected in the inner part of the PSPC images.

\section{The VLA FIRST Survey}

FIRST (Faint Images of the Radio Sky at Twenty-cm) is a project designed to produce the radio equivalent of the Palomar Observatory Sky Survey (Becker et al. 1995, White et al. 1997) using the NRAO Very Large Array.

The catalogue contains $\sim 138,000$ sources produced from the 1993 and 1994 images. The astrometric reference frame of the maps is accurate to $0.05^{\prime \prime}$. Approximately $15 \%$ of the sources have optical counterparts at the limit of the POSS I plates; unambiguous optical identifications are achievable to $m_{v} \sim 24$. The survey area has been chosen to coincide with that of the Sloan Digital Sky Survey; at its $m_{v} \sim 24$ limit, $\sim 50 \%$ of the optical counterparts to FIRST sources will be detected. 


\section{The FIRST Radio Counterparts of WGACAT Sources}

2,300 objects, all the WGACAT detections in the inner part of the PSPC images, within the area were selected. 151 of these sources have a FIRST counterpart and only 68 of them have optical identifications. Most of the FIRST sources are not detected in the ROSAT images. Redshifts are available for 44 associations. The classification of the 68 WGACAT/FIRST associations with optical identifications is given in Table 1.

TABLE 1. Classification of the 68 WGACAT/FIRST associations with available optical identifications

\begin{tabular}{ccccccc}
\hline Galaxies & QSOs & BL-Lacs & AGN & Clust. of galax. & Stars & Radio Galaxies \\
\hline 28 & 17 & 8 & 8 & 4 & 2 & 1 \\
\hline
\end{tabular}

\section{Radio Morphology}

The radio counterparts of 111 of the WGACAT sources are single sources, while 23 are double, 11 are triple and 6 have four or more radio components.

Figure 1 shows as an example the optical DSS, the FIRST radio, and the ROSAT images. The optical counterpart is the faint object close to the X-ray position and to the central radio component. This is probably a distant radio galaxy.

\section{Optical Follow-up of the WGACAT/FIRST Sources}

Since the WGACAT catalogue contains sources from a large number of ROSAT images, and most of these lack complete optical identifications, the analysis of the WGACAT/FIRST associations is strongly limited. We plan to complete the optical identification of complete samples. The objects identified previously come from relatively bright sources, by studying the fainter population we expect to find larger and complete samples of X-ray objects with radio counterparts at higher redshifts. The current highest redshift in our WGACA/FIRST sample is 2.334

\section{References}

Becker, R. et al. 1995, Astrophys.J. 450, 559

White, N.E. et al. 1994, IAU Circ. No.6100

White, R.L. et al. 1997, Astrophys.J. in press 


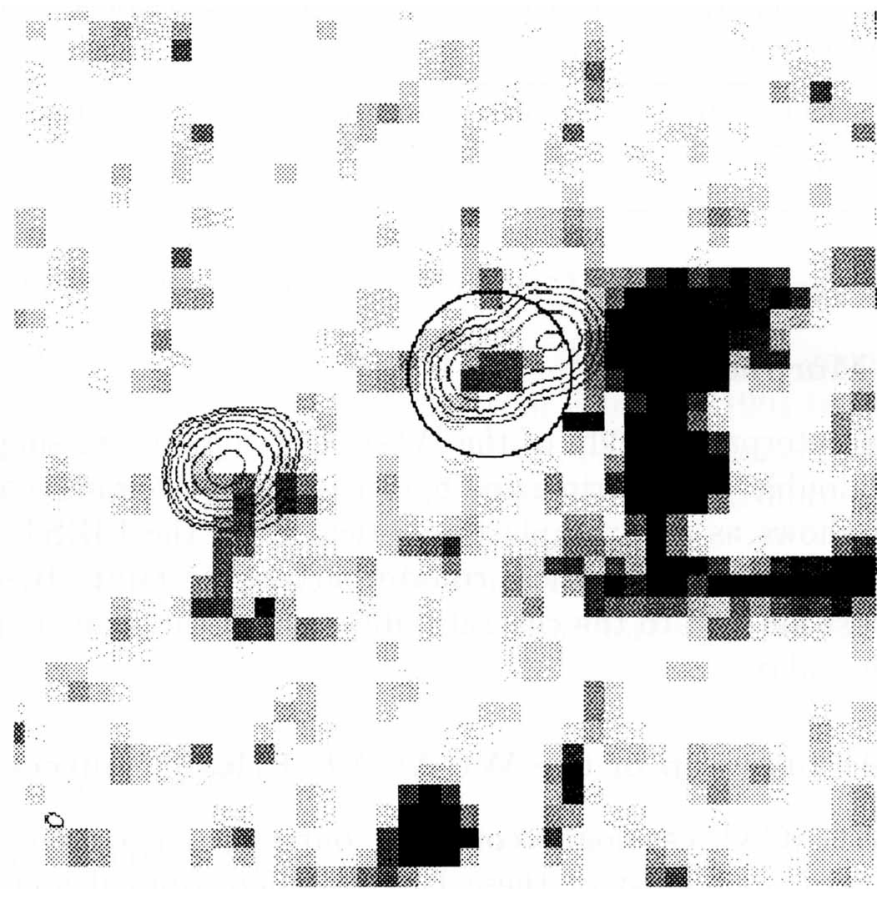

Figure 1. Optical (image), radio (contours), and X-ray data (error circle of radius $\sim 6.5$ $\operatorname{arcsec}$ ) of one of the radio-loud X-ray selected sources of the ROSAT sample. 